\section{AB0635 ANTI-PHOSPHOLIPID ANTIBODIES IN LUPUS MYOCARDITIS}

V. Dhir ${ }^{1}$, A. Gawalkar ${ }^{1}$, J. Ahluwalia ${ }^{2}$, A. Bahl ${ }^{3}$, S. Sharma ${ }^{1}$, A. Sharma ${ }^{1}$, S. Jain ${ }^{1}$. ${ }^{1}$ Internal Medicine (Rheumatology Unit); ${ }^{2}$ Hematology; ${ }^{3}$ Cardiology, Post Graduate Institute of Medical Education and Research, Chandigarh, India

Background: Myocarditis in lupus is an uncommon clinical manifestation, with unknown pathogenesis. ${ }^{1}$ Suggested etiologies includes Immune-complexes, cell mediated damage and anti-phospholipid antibodies. The latter may affect the myocardial function microthrombi in cardiac vessels or direct cytotoxicity. $\left(^{2}\right.$ Previously, small studies have suggested an association between antiphospholipid tests as myocarditis.

Objectives: To evaluate whether myocarditis in SLE is associated with antiphospholipid positivity.

Methods: This was a cross-sectional study in which patients fulfilling SLICC criteria 2012 for SLE or Alarcon Segovia criteria for MCTD were included after consent. Patients were recruited as 'Cases' if they had myocarditis/cardiomyopathy defined by poor generalised contractility and/or dilation of all chambers nad/or reduced ejection fraction on echocardiography without any obvious cause. Those with regional wall motion abnormalities or pulmonary artery hypertension (moderate or severe) were excluded. Controls were age ( \pm 2.5 years) and disease duration $( \pm 25 \%)$ matched patients of SLE without any abnormality on echocardiography. Serum titers of anticardiolipin antibodies and b2 GP1 (both IgG and IGM) were measured by commercial ELISA kit. Lupus anticoagulant was detected by Dilute Russell Viper Venom Test (dRVVT) with both screening (prolonged) and confirmation steps (shortening on higher phospholipid content, ratio $>1.2$ ) on doubly centrifuged, platelet poor plasma. Proportions were compared using chi-square test (or Fischers exact test) and continous variables by MannWhitney $U$ test.

Results: A total of 51 patients were recruited in this study that included 21 cases and 30 controls. All had SLE, except 1 case was of MCTD (among cases). There was no difference in mean $( \pm S D)$ age $(33.3 \pm 14.7,32.8 \pm 12.4$ years, $p=0.9)$ or median (interquartile range) disease duration $\left(30^{8-38} 25\right.$ (13.5-45) months, $\mathrm{p}=0.6$ ) between groups. Mean ejection fraction of Cases was $31.7 \%( \pm 9.3 \%)$ while that of Controls was $55.7 \%( \pm 1.7 \%)(p<0.001)$. There were no significant differences between proportion of Cases (42.9\%) and Controls (40\%) with positive antiphospholipid tests $(p=0.7)$. The majority had positive anticardiolipin antibodies, followed by b2 GP1, and lupus anticoagulant was positive in only two in each group (figure 1). Among 9 Cases positive at baseline, 6 patients could be re-tested (2 expired and 1 was lost), out of which 3 had persistent positivity. Out of 12 Controls positive once, 8 could be retested ( 1 expired and 3 were lost), with 1 being persistently positive. There was no signficiant difference in persistent positivity between groups. $(p=0.3)$.

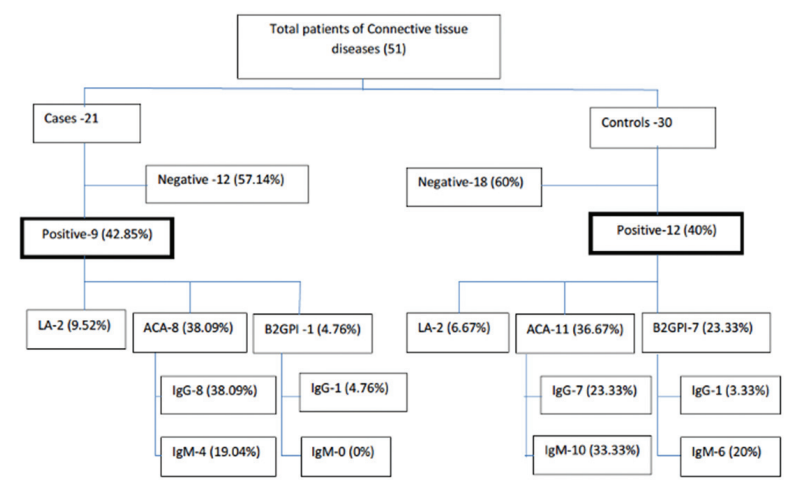

Abstract AB0635 - Figure 1

Conclusions: This study did not find any signficant association between antiphospholipid antibodies (single time or persistent) with cases of lupus myocarditis.

\section{REFERENCES:}

[1] Doria A, laccarino L, Sarzi-Puttini P, Atzeni F, Turriel M, Petri M. Cardiac involvement in systemic lupus erythematosus. Lupus. 2005;14(9):683-6.

[2] Bidani AK, Roberts JL, Schwartz MM, Lewis EJ. Immunopathology of cardiac lesions in fatal systemic lupus erythematosus. The American journal of medicine. 1980;69(6):849-58.

Disclosure of Interest: None declared

DOI: 10.1136/annrheumdis-2018-eular.3672

\section{$\mathrm{AB} 0636$ \\ DAMAGE ACCRUAL IN SYSTEMIC LUPUS ERYTHEMATOSUSIS NOT RELATED TO SUSTAINED HYPOCOMPLEMENTEMIA}

W.D. Raymond $^{1}$, L. Tyack ${ }^{1}$, G. Ø. Eilertsen ${ }^{2}$, J. Nossent ${ }^{1} .{ }^{1}$ School of Medicine and Pharmacology, University of Western Australia, Perth, Australia; ${ }^{2}$ Molecular Inflammation Research Group, Department of Clinical Medicine, Arctic University, Tromso, Norway

Background: Hypocomplementemia $(\mathrm{HC})$ represents a significant clinical finding in Systemic Lupus Erythematosus (SLE) as it suggests complement activation by immune-complexes, which can initiate inflammation. As disease activity contributes to damage accrual in SLE patients, we investigated the role of $\mathrm{HC}$ as a pre dictor of subsequent organ damage.

Objectives: Investigate the relationship between $\mathrm{HC}$, disease flares and organ damage accrual in SLE patients.

Methods: Longitudinal cohort study of 102 SLE patients with $\mathrm{HC}$ defined as a C3 and/or C4 levels below cut-off during median follow-up of 13.8 years (IQR 7.0 23.1). Disease activity was scored by time averaged SLEDAI-2K without the serological components (cWAS), flares by SELENA-SLEDAI and damage accrual by SLICC-DI. Analysis included comparisons between normocomplementemic (NC) and hypocomplementemic $(\mathrm{HC})$ patients, and multivariate logistic and Cox regres sion modelling determined the predictive value of $\mathrm{HC}$ on organ damage.

Results: $\mathrm{HC}$ occurred in $2 / 3$ of patients overall and was more often due to low $\mathrm{C} 3$ (97\%) than low C4 (54\%). HC patients had a higher prevalence of anti-dsDNA Ab (72\% vs $36 \%, \mathrm{p}<0.01)$ and $\mathrm{aPL}(74 \%$ vs $40 \%, \mathrm{p}<0.01)$, but $\mathrm{HC}$ concurred with anti-dsDNA presence in only $36 \%$ of cases. HC patients had higher maximum cSLEDAI scores, but the time adjusted cWAS scores (1.9 vs $1.2, p=0.9)$ and the frequency and risk of overall damage accrual $(S D I>0, n=60)$ associated with $\mathrm{HC}$ was similar as for NC patients (OR 1.08, p>0.20).

Conclusions: Low complement levels occur in 2/3 of SLE patients but have negligible impact on time averaged disease activity and damage accrual in SLE. Dis crepancies between low $\mathrm{C} 3$, low $\mathrm{C} 4$ and anti-dsDNA Ab occurrence indicate that in SLE alternative complement activation occurs frequently and requires furthe translational study.

Acknowledgements: The Rheumatology Group of UWA (JN) was supported by an unrestricted grant from the Arthritis and Osteoporosis Foundation of Western Australia (AOWA). The AOWA provided WR with a PhD Scholarship in Memory of Johan Donald Stewart.

Disclosure of Interest: None declared

DOI: 10.1136/annrheumdis-2018-eular.1643

\section{AB0637 1 HAEMATOLOGICAL INVOLVEMENT OF PRIMARY SJOGREN'S SYNDROME PATIENTS IN A SINGLE CENTRE STUDY OF 232 CHINESE CASES}

X. Wang, Z. Wu, Y. Liang, F. Cheng, R. Feng, X. Fang, S. Yang, B. Gong, J. Tang. Department of Rheumatology, Tongji Hospital, Tongji University School of Medicine, Shanghai, China

Background: Primary Sjögren's syndrome (pSS) is a commono systemic auto immune disease, characterised by lymphocytic infiltration of the secretory glands and different extraglandular manifestations. Besides haematological disorders are prevalent but not well recognised in patients with pSS.

Objectives: Our aim is to determine the existence of cytopenia at diagnosis or during follow-up of our pSS patients as well as the associated factors.

Methods: A cohort of pSS patients that had been followed-up in the Department of Rheumatology, Tongji hospital of Tongji University, from 2011 to 2017 was retrospectively assessed. Clinical and laboratory findings about the patients were recorded

Results: Out of 232 pSS patients composing the cohort, cytopenia was already present in $55.60 \%(n=129)$ at the time of diagnosis. Anaemia was detected in $30.17 \%(n=70)$, leukopenia in $37.5 \%(n=87)$, neutropenia in $22.41 \%(n=52)$, and thrombocytopenia in $26.72 \%$ ( $n=62)$ of patients. The proportion of patients with cumulative cytopenia was $6.47 \%(n=15)$. Cumulative cytopenia was diseaserelated in $5.60 \%(n=13)$ and medication-related in $0.86 \%(n=2)$ of the patients. In patients with cytopenia at the time of diagnosis, erythrocyte sedimentation rates (ESR) were higher $(\mathrm{p}<0.001), \mathrm{C} 3$ and C4 hypocomplementemia was more prevalent $(p<0.001, p=0.060)$, and they were positive for anti-SSB at a greater proportionand $(p=0.058)$. Hyperglobulinemia, C3 hypocomplementemia, positive antiSSA and anti-SSB might increase the incidence of anaemia in pSS, with OR of $2.700,2.042,1.537$ and 1.901, respectively. In addition, logistic regression analysis suggest C3 hypocomplementemia might be associated with different types Leukopenia $(p<0.01)$, while abnormal Transaminase and C4 hypocomplementemia are independent risk factors for thrombocytopenia with a OR of 4.171 (1.516$11.480)$ and 5.697 (1.662-19.523). Cytopenia in pSS patients not either at diagnosis or during follow-up, was always easy to be ameliorated, but several cases leaded to unfavourable outcome. 